\title{
Laparoscopic Repair of Perforated Peptic Ulcer: Outcome and Associated Morbidity and Mortality
}

Mahdi Alemrajabi ${ }^{1}$, Saeed Safari ${ }^{2}$, Adnan Tizmaghz ${ }^{3}$, Fatemeh Alemrajabi ${ }^{4}$, Ghazaal Shabestanipour ${ }^{5}$

${ }^{1}$ MD., Assistant Professor of Colorectal Surgery, Firoozgar Clinical Research Development Center (FCRDC), Iran University of Medical Sciences, Tehran, Iran

${ }^{2}$ MD., Assistant Professor of General Surgery, Firoozgar Clinical Research Development Center (FCRDC), Iran University of Medical Sciences, Tehran, Iran

${ }^{3}$ MD., Resident in General Surgery, Iran University of Medical Sciences, Tehran, Iran

${ }^{4}$ MD., Resident of Anesthesiology, Yazd University of Medical Science, Yazd, Iran

${ }^{5}$ MD., General Physician, Shahid Beheshti University of Medical Sciences, Tehran, Iran

\section{Type of article: Short report}

\begin{abstract}
Introduction: The mainstay of treatment for perforated peptic ulcer is Omental patch closure. With the advent of laparoscopic surgery, this approach is being used for the treatment of perforated peptic ulcer. The aim of this study was to evaluate the outcome of laparoscopy in Firoozgar general hospital over a period of 18 months. The outcome of the laparoscopic approach and the associated morbidity and mortality, operation time, conversion rate and hospital stay were assessed.

Methods: A prospective analysis of 29 consecutive patients (mean age 37.5 years; 23 men) with perforated peptic ulcers and who had undergone laparoscopic surgery was carried over a period of 18 months from March 2014 until September 2015. Pre-operative, intra-operative, and post-operative clinical data were collectively analyzed by SPSS 19 for Windows.

Results: Seventeen patients had a history of cigarette smoking, 11 patients had a history of opium consumption, 19 were chronic NSAID users, 26 had Helicobacter pylori infections, and six had a co-morbid condition. Previous surgical history included laparotomy for pancreatic cancer in two patients, for sigmoid colon cancer in one patient, and for acute appendicitis in four patients. The average operating time for all cases was $47.5+20$ min. The mean lag time between onset of symptoms and surgery was 20.4 hours. All patients underwent laparoscopic closure of the perforation with Omental patch closure. No morbidity was observed, and none of the patients needed conversion to open surgery. One patient died after 11 months of follow-up due to the progression of underlying pancreatic cancer. The mean postoperative hospital stay was 4.2 days.

Conclusions: The results of the laparoscopic approach for perforated peptic ulcer were promising, with no conversion to open surgery, no morbidity, and mortality.

Keywords: perforated peptic ulcer; laparoscopic repair, outcome
\end{abstract}

\section{Introduction}

Perforated peptic ulcer is the most common indication for emergency gastric surgery. It is associated with high morbidity and mortality (1). The peak incidence of complications associated with peptic ulcer disease between the seventh and eighth decades of life. It is encountered more frequently in males $(\mathrm{M}: \mathrm{F}=1.5: 1)$ (2). Omental patch closure is the mainstay of treatment for perforated peptic ulcer $(3,4)$. With the advent of laparoscopic surgery, this approach is being used for the treatment of perforated peptic ulcer. Skill in laparoscopic abdominal emergencies is needed (5). The current study aimed to evaluate the outcome of laparoscopy in Firoozgar general hospital over a period of 20 months. The outcome of the laparoscopic approach and the associated morbidity and mortality, operation time, conversion rate, and length of hospital stay were assessed.

\section{Corresponding author:}

Assistant Professor Dr. Saeed Safari, Firoozgar Clinical Research Development Center (FCRDC), Iran University of Medical Sciences, Tehran, Iran. Tel: +98.9126053406, Email: saeedsfr@gmail.com

Received: January 01, 2016, Accepted: April 10, 2016, Published: June 2016

iThenticate screening: March 04, 2016, English editing: May 10, 2016, Quality control: June 04, 2016

(C) 2016 The Authors. This is an open access article under the terms of the Creative Commons Attribution-NonCommercialNoDerivs License, which permits use and distribution in any medium, provided the original work is properly cited, the use is non-commercial and no modifications or adaptations are made. 


\section{Material and Methods}

A prospective analysis of 29 consecutive patients (mean age 37.5 years; 23 men) with perforated peptic ulcer who had undergone laparoscopic surgery was conducted over a period of 18 months from March 2014 until September 2015. All procedures were performed after appropriate fluid infusion and administration of appropriate antibiotics (Ceftizoxime + Metronidazol) by the same surgeon. All of the patients were anticoagulated with the injection of subcutaneous, unfractionated heparin (500 IU) before the procedure. In summary, a 30-degree reverse Trendelenburg position was used for the patients. The surgeon stood at the feet of the patient. The first periumbilical trocar was placed by using the Hasson technique. A pneumoperitoneal pressure of 12-14 mmHg was applied in most cases. An angled "Storz" laparoscope (30 degrees) was introduced into the abdomen through the $11-\mathrm{mm}$ trocar to inspect the intra-abdominal organs, including the surface of the liver, gallbladder, stomach, intestine, pelvic organs, and visible retroperitoneal surfaces, along with examination for free intraperitoneal fluid. A second 11-mm trocar was placed at the right upper abdomen, and another 5-mm trocar was placed at the left upper abdomen. Closing the perforated ulcer was done by using two separate $2 / 0$ Vicryl sutures. The omental flap was mobilized from the greater omentum over the sutured ulcer and tied over by previous retained suture. The peritoneal cavity was washed out with warm saline until the area was cleared in all patients. Statistical analysis of the data was done by SPSS 19 for Windows (SPSS Inc., Chicago, Illinois, USA).

\section{Results}

Seventeen patients (58\%) had a history of cigarette smoking, 11 patients $(37 \%)$ had a history of opium consumption, $19(65 \%)$ were chronic NSAID users, $26(89 \%)$ had Helicobacter pylori infection, and six had a co-morbid condition. Previous surgical history included previous laparotomy for pancreatic cancer in two patients, for sigmoid colon cancer in one patient, and for acute appendicitis in four patients. The average operating time for all cases was $47.5+20 \mathrm{~min}$. The mean lag time between onset of symptoms and surgery was 20.4 hours. All patients underwent laparoscopic closure of the perforation with omental patch closure, and a Silicone Pezzer Drain was inserted at the site of the perforation. No morbidity was observed and no patient needed conversion to open surgery. The nasogastric tube was removed 24 hours after surgery in all 29 cases, and liquid diet was started on the second postoperative day, and it was advanced to regular diet on the third post-operative day. The Pezzer Drain was removed on the fourth post-operative day. One patient died after 11 months of follow-up due to the progression of underlying pancreatic cancer. The mean post-operative hospital stay was 4.2 days [four days (22 patients), six days (five patients), and seven days (two patients)].

\section{Discussion}

The first description of laparoscopic approach of perforated peptic ulcer was made by P. Mouret et al. in 1990 (6). Since then, there have been numerous reports on the benefits of the laparoscopic procedure versus the open procedure $(7,8)$. Also, a few reports concluded that the laparoscopic treatment does not show additional benefits over the open procedure $(3,8)$. W.Y. Lauet al.'s study indicated that laparoscopic repair takes longer than the open procedure but reduces the post-operative consumption of analgesics (8). We didn't measure post-operative pain in our patients because 11 patients had history of opium consumption, and 19 were chronic NSAID users. In our study, the average operating time for all cases was $47.5 \pm 20$ minutes. In Alwadaani's study in Saudi Arabia, the mean operating time was $42 \pm 16.7$ minutes, which was shorter than our study and other reports in the literature (9). This could have been due to the shorter irrigation time in Alwadaani's study. The decreasing incidence of peptic ulcers has reduced the use of laparoscopic repair for this condition (10). As a result, we only had 29 patients with perforated peptic ulcers during the 20 -month period.

\section{Conclusions}

Currently, most young surgeons as well as experienced surgeons believe that the laparoscopic technique can replace most open surgeries for many abdominal pathologies. Our study demonstrated promising results for the laparoscopic approach for perforated peptic ulcer, with no conversion to open surgery, no morbidity, and no mortality. But, we recommend more prospective studies and clinical trials with larger numbers of cases to establish the benefits of laparoscopic repair of perforated peptic ulcer over open repair.

\section{Acknowledgments:}

We thank all of the participants in this study for generously helping us in this research. We also thank all of the surgical residents, nurses, and other practice staff at Firoozgar Hospital who kindly assisted us by their care of the patients. 


\section{Conflict of Interest:}

There is no conflict of interest to be declared.

\section{Authors' contributions:}

All authors contributed to this project and article equally. All authors read and approved the final manuscript.

\section{References:}

1) Guadagni S, Cengeli I, Galatioto C, Furbetta N, Piero VL, Zocco G, et al. Laparoscopic repair of perforated peptic ulcer: single-center results. Surg Endosc. 2014; 28(8): 2302-8. doi: 10.1007/s00464-014-3481-2. PMID: 24609709.

2) Wang YR, Richter JE, Dempsey DT. Trends and outcomes of hospitalizations for peptic ulcer disease in the United States, 1993 to 2006. Ann Surg. 2010; 251(1): 51-8. doi: 10.1097/SLA.0b013e3181b975b8. PMID: 20009753.

3) Siu WT, Leong HT, Li MKW. Single stitch laparoscopic omental patch repair of perforated peptic ulcers. J R Coll Surg Edinb. 1997; 42: 92-4. PMID: 9114677.

4) Katkhouda N, Mavor E, Mason RJ, Campos GM, Soroushyari A, Berne TV. Laparoscopic repair of perforated duodenal ulcers, Outcome and efficacy in 30 consecutive patients. Arch Surg. 1999; 134: 84550. doi: 10.1001/archsurg.134.8.845. PMID: 10443807.

5) Paterson-Brown S. Emergency laparoscopic surgery. $\mathrm{Br} \quad \mathrm{J}$ Surg. 1993; 80: 279-83. doi: 10.1002/bjs.1800800305. PMID: 8472132.

6) Mouret P, François Y, Vignal J, Barth X, Lombard-Platet R. Laparoscopic treatment of perforated peptic ulcer. Br J Surg. 1990; 77(9): 1006. PMID: 2145052.

7) Robertson GS, Wemyss-Holden SA, Maddern GJ. Laparoscopic repair of perforated peptic ulcers, The role of laparoscopy in generalized peritonitis. Ann R Coll Surg Engl. 2000; 82(6): 814-6. PMID: 10700758, PMCID: PMC2503463.

8) Lau WY, Leung KL, Zhu XL, Lam YH, Chung SC, Li AK. Laparoscopic repair of perforated peptic ulcer. Br J Surg. 1995; 82 (6): 814-6. doi: 10.1002/bjs.1800820630. PMID: 7627519.

9) Al Wadaani H. Emergent laparoscopy in treatment of perforated peptic ulcer: a local experience from a tertiary centre in Saudi Arabia. World J Emerg Surg. 2013; 8: 10. doi: 10.1186/1749-7922-8-10. PMID: 23497473, PMCID: PMC3614460.

10) Bashinskaya B, Nahed BV, Redjal N, Kahle KT, Walcott BP. Trends in peptic ulcer disease and the identification of Helicobacter pylori as a causative organism: Population-based estimates from the US nationwide inpatient sample. J Glob Infect Dis. 2011; 3: 366-70. doi: 10.4103/0974-777X.91061. PMID: 22224001, PMCID: PMC3249993. 